\title{
MicroRNA-based therapy and breast cancer: a comprehensive review of novel therapeutic strategies from diagnosis to treatment
}

\begin{abstract}
MicroRNAs (miRNA) are 21-23 nucleotide molecules not translated into proteins that bind and target the 3' untranslated regions of mRNA. These characteristics make them a possible tool for inhibiting protein translation. Different cellular pathways involved in cancer development, such as cellular proliferation, apoptosis, and migration, are regulated by miRNAs. The objective of this review is to discuss various miRNAs involved in breast cancer in detail as well as different therapeutic strategies from the clinic to industry. A comprehensive discussion is provided on various miRNAs involved in breast cancer development, progression, and metastasis as well as the roles, targets, and related therapeutic strategies of different miRNAs associated with breast cancer. miRNAs known to be clinically useful for the diagnosis and prognosis of breast cancer are also discussed. Different strategies and challenges, including nucleic acid-based (miRNA mimics, antagomiRs, and miRNA sponges) and drug-based (drug resistance, drugs/miRNA interaction, nanodelivery, and sensing systems) approaches to suppress specific oncogenes and/or activate target tumor suppressors are discussed. In contrast to other articles written on the same topic, this review focuses on the therapeutic and clinical value of miRNAs as well as their corresponding targets in order to explore how these strategies can overcome breast cancer, which is the second most frequent type of cancer worldwide. This review focuses on promising and validated miRNAs involved in breast cancer. In particular, two miRNAs, miR-21 and miR-34, are discussed as the most promising targets for RNA-based therapy in non-invasive and invasive breast cancer, respectively. Finally, relevant and commercialized therapeutic strategies are highlighted.
\end{abstract}

Keyword: miRNA; Apoptosis; Oncogene; Tumor suppressor; Drug resistance; Breast cancer; Therapy 